\title{
Clinical experience with 12-month follow-up in patients after implantation of a novel long-tapered sirolimus drug-eluting stent
}

\author{
Jakub Podolec ${ }^{1}$, Maciej Skubera², Łukasz Niewiara ${ }^{1}$, Mateusz Podolec $^{3}$, Piotr Pieniążek ${ }^{1}$, Krzysztof Bartuśs $^{4}$, \\ Krzysztof Żmudka' ${ }^{1}$ Jacek Legutko ${ }^{1}$
}

1Department of Interventional Cardiology, Jagiellonian University College of Medicine, John Paul II Hospital, Krakow, Poland ${ }^{2}$ Department of Cardiac and Vascular Diseases, Jagiellonian University College of Medicine, John Paul II Hospital, Krakow, Poland ${ }^{3}$ Department of Coronary Artery Disease, Jagiellonian University College of Medicine, John Paul II Hospital, Krakow, Poland ${ }^{4}$ Department of Cardiovascular Surgery and Transplantology, Jagiellonian University College of Medicine, John Paul II Hospital, Krakow, Poland

\begin{abstract}
A bstract
Introduction: Long lesions contribute to a significant number of percutaneous coronary interventions.

Aim: To assess the efficacy and safety of a novel long-tapered drug-eluting stent (DES) at a 12-month follow-up (FU) in patients with long coronary atherosclerotic lesions

Material and methods: A prospective clinical cohort study was conducted in 32 patients who underwent percutaneous coronary intervention using a BioMime Morph tapered stent (Meril Life Sciences, India). The patients were followed for 3, 6, and 12 months. The safety endpoints were death, myocardial infarction (MI), target lesion revascularization (TLR), target vessel revascularization (TVR), and MACE and/or major bleeding.

Results: Mean lesion length was $48 \mathrm{~mm}$ (range: $35-70 \mathrm{~mm}$ ) measured via quantitative coronary analysis (QCA). In most cases, the target lesion was located in the LAD (68.75\%). A GuideLiner catheter (Vascular Solutions Inc., MN, USA) was used in $12.5 \%$ of procedures; buddy-wire technique in $9.4 \%$ of cases. Bifurcation lesions were treated in $40.6 \%$ of cases. Additional stent implantation was needed in $56 \%$ of the procedures ( $25 \%$ of cases due to proximal or distal dissection, or due to insufficient stent length in $31 \%$ of cases). On 12-month FU we observed 1 TLR (3.1\%), 1 TVR (3.1\%), and 1 non-cardiovascular death.

Conclusions: The long sirolimus-eluting stent with tapered structure was characterized by good deliverability in long coronary lesions, although in some cases "buddy wire" or extension microcatheter use was necessary. Follow-up at 3, 6, and 12 months showed no significant major adverse cardiovascular events related to the device.
\end{abstract}

Key words: long-tapered stent, drug-eluting stent, long coronary lesion, coronary artery disease.

S u m m a ry

The long sirolimus-eluting stent with tapered structure was characterized by good deliverability in long coronary lesions, although in some cases "buddy wire" or extension microcatheter use was necessary. Previous lesion preparation even with non-compliant balloon is important for successful stent introduction. Operator experience plays an important role. Follow-up at 3, 6, and 12 months showed no significant major adverse cardiovascular events related to the device.

\section{Introduction}

Atherosclerosis is a progressive inflammatory disease and among the aging population becomes a challenge for interventional cardiologists [1-3]. Percutaneous treatment is recommended in most patients with significant coro- nary artery disease (CAD) [4]. Long lesions contribute to a significant number of percutaneous coronary interventions $(\mathrm{PCl})$, which requires the use of multiple overlapping stents, mostly in the context of insufficient stent length or challenging deliverability of longer stents [5]. However,

\section{Corresponding author:}

Jakub Podolec MD, PhD, Department of Interventional Cardiology, Jagiellonian University College of Medicine, John Paul II Hospital, 80 Pradnicka St, 31-202 Krakow, Poland, e-mail: jjpodolec@gmail.com

Received: 15.12.2018, accepted: 7.01.2019. 
the strategy of using overlapping stents can result in stent misplacement as well as increased risk of restenosis [5]. The newer-generation drug-eluting stents (DES) are designed with reduced strut thickness and have been shown to be efficacious in treating very long coronary lesions $[6,7]$. BioMime Morph (Meril Life Sciences Pvt. Ltd., Vapi, India) is a long-tapered sirolimus-eluting stent (SES) system designed for the treatment of very long coronary lesions including $\mathrm{PCl}$ of chronic total occlusions [6]. This long-tapered stent has been demonstrated to be associated with good procedural and 1-year clinical outcomes in complex patients with long and diffuse lesions [8].

\section{Aim}

The aim of our study was to assess clinical aspects, efficacy, and short-term follow-up safety of the novel long DES with tapered structure, tailored to fit the coronary anatomy in patients with long coronary atherosclerotic lesions.

\section{Material and methods}

In this prospective clinical cohort study, we included 32 consecutive patients admitted to the Department of Interventional Cardiology, Jagiellonian University College of Medicine and the John Paul II Hospital, Krakow, Poland between May 2016 and July 2017, who underwent PCI and in whom implantation of a long, tapered DES was performed due to the presence of coronary lesions longer than $35 \mathrm{~mm}$. BioMime Morph is an open-cell cobalt-chromium sirolimus-eluting stent with closed cells on its tapered end and was implanted in all patients who qualified for inclusion in the study. The $\mathrm{PCl}$ procedures were

Table I. Clinical characteristics of patients and procedures

\begin{tabular}{|c|c|}
\hline Risk factors & Value \\
\hline Male sex & $22(69 \%)$ \\
\hline Age, mean \pm SD [years] & $68.0 \pm 8.0$ \\
\hline BMI, mean $\pm S D\left[\mathrm{~kg} / \mathrm{m}^{2}\right]$ & $29.6 \pm 4.1$ \\
\hline \multicolumn{2}{|l|}{ Medical history: } \\
\hline Arterial hypertension & $30(93.8 \%)$ \\
\hline Systolic blood pressure, mean \pm SD [mm Hg] & $131 \pm 15$ \\
\hline Diabetes & $11(34.4 \%)$ \\
\hline Impaired glucose tolerance & $4(12.5 \%)$ \\
\hline Dyslipidemia & $28(87.5 \%)$ \\
\hline Prior $\mathrm{PCl}$ & $14(45.2 \%)$ \\
\hline Prior $\mathrm{CABG}$ & $2(6.3 \%)$ \\
\hline Prior myocardial infarction & $14(43.8 \%)$ \\
\hline \multicolumn{2}{|l|}{ Indication for invasive coronary angiography: } \\
\hline Stable coronary artery disease & $27(84.4 \%)$ \\
\hline Non-ST elevation myocardial infarction & $3(9.4 \%)$ \\
\hline ST elevation myocardial infarction & $1(3.1 \%)$ \\
\hline Unstable coronary artery disease & $1(3.1 \%)$ \\
\hline \multicolumn{2}{|l|}{ Artery treated: } \\
\hline Left anterior descending & $22(68.8 \%)$ \\
\hline Left circumflex & $4(12.5 \%)$ \\
\hline Right coronary artery & $6(18.8 \%)$ \\
\hline Bifurcation lesions & $13(40.6 \%)$ \\
\hline MEDINA $(0,1,1)$ & $3(9.4 \%)$ \\
\hline MEDINA $(1,0,1)$ & $1(3.1 \%)$ \\
\hline MEDINA $(1,1,0)$ & $5(15.6 \%)$ \\
\hline MEDINA $(1,1,1)$ & $4(12.5 \%)$ \\
\hline
\end{tabular}

Risk factors

Value

Implanted stent length:

\begin{tabular}{lc}
\hline $40 \mathrm{~mm}$ & $10(31.3 \%)$ \\
\hline $50 \mathrm{~mm}$ & $13(40.6 \%)$ \\
\hline $60 \mathrm{~mm}$ & $9(28.1 \%)$
\end{tabular}

Implanted stent diameter:

\begin{tabular}{lc}
\hline $2.75-2.25 \mathrm{~mm}$ & $6(18.8 \%)$ \\
\hline $3.0-2.5 \mathrm{~mm}$ & $18(56.3 \%)$ \\
\hline $3.5-3.0 \mathrm{~mm}$ & $7(21.9)$ \\
\hline $4.0-3.5 \mathrm{~mm}$ & $1(3.1 \%)$
\end{tabular}

Procedure information:

\begin{tabular}{lc}
\hline NC balloon pre-dilatation & $8(25.0 \%)$ \\
\hline Stiff guiding wire used & $11(34.4 \%)$ \\
\hline "Buddy-wire" technique & $3(9.4 \%)$ \\
\hline GuideLiner microcatheter use & $4(12.5 \%)$ \\
\hline Additional stent needed & $18(56.3 \%)$ \\
\hline Dissection present & $8(25.0 \%)$ \\
\hline Dissection proximal to first stent & $3(9.4 \%)$ \\
\hline Dissection distal to first stent & $5(15.6 \%)$ \\
\hline Stent length too short & $10(31.3 \%)$ \\
\hline Post-dilatation NC balloon use & $22(68.8 \%)$ \\
\hline IVUS used & $2(6.3 \%)$ \\
\hline Final TIMI 3 flow & $32(100.0 \%)$ \\
\hline Contrast medium, median (IQR) [ml] & $260(200-370)$ \\
\hline Radiation dose, median (IQR) [mGy] & $1597(761-2941)$
\end{tabular}

$B M I$ - body mass index, $S D$ - standard deviation, $D_{\max }$ - maximal diameter measured in $Q C A, P C I$ - percutaneous coronary intervention, $C A B G$ - coronary artery bypass grafting, IQR - interquartile range. 
performed according to current practice guidelines [4]. All patients provided written informed consent prior to the procedure. Interventional and pharmacological strategies were left to the discretion of the operators. Distal-maximal and proximal-maximal diameter $\left(\mathrm{D}_{\max }\right)$ were calculated via quantitative coronary analysis (QCA) as diameters of the vessel on the proximal and distal edge of the baseline lesion, respectively. Optimal PCI result was defined as full lesion coverage without coronary artery dissection and with proper apposition of the stent (less than $20 \%$ residual stenosis) in two perpendicular angiographic projections. The clinical outcomes were assessed at 3-month, 6-month, and 12-month follow-ups.

At the 12-month observation, the following endpoints were assessed: death, myocardial infarction, target lesion revascularization (TLR), target vessel revascularization (TVR), and major cerebrovascular event and/or major bleeding, adjudicated by independent researchers blinded to patient characteristics. Major bleeding was defined as bleeding with a need for hospitalization and/ or was fatal to the patient. All study procedures were performed according to local bioethics committee guidance as well as the Helsinki Declaration.

\section{Statistical analysis}

We expressed continuous variables as mean with standard deviation or median with interquartile range for non-normal distributions. Discrete variables were pre-

Table II. QCA analysis of coronary lesions

\begin{tabular}{|c|c|}
\hline QCA analysis & Value \\
\hline \multicolumn{2}{|l|}{ Prior to angioplasty: } \\
\hline MLD prior to $\mathrm{PCl}$, mean (SD) [mm] & $0.68(0.25)$ \\
\hline Percent diameter stenosis, mean (SD) [\%] & $78.0(13.6)$ \\
\hline Lesion length, median (min.-max.) [mm] & $50.93(38.4-80.1)$ \\
\hline Reference diameter & $2.65(0.34)$ \\
\hline $\mathrm{D}_{\max }$ proximal, mean (SD) $[\mathrm{mm}]$ & $2.97(0.30)$ \\
\hline $\mathrm{D}_{\max }$ distal, mean (SD) [mm] & $2.16(0.49)$ \\
\hline \multicolumn{2}{|l|}{ After angioplasty: } \\
\hline MLD after $\mathrm{PCl}$, mean (SD) [mm] & $2.37(0.45)$ \\
\hline Residual stenosis after $\mathrm{PCl}[\%]$ & $9.1(9.9)$ \\
\hline $\mathrm{D}_{\max }$ proximal, mean (SD) $[\mathrm{mm}]$ & $3.13(0.38)$ \\
\hline $\mathrm{D}_{\max }$ distal, mean (SD) [mm] & $2.34(0.46)$ \\
\hline $\begin{array}{l}\text { Proximal stent to vessel lumen diameter } \\
\text { ratio, mean (SD) }\end{array}$ & $0.99(0.07)$ \\
\hline $\begin{array}{l}\text { Distal stent to vessel lumen diameter ratio, } \\
\text { mean (SD) }\end{array}$ & $1.14(0.18)$ \\
\hline Lesion length to stent length ratio, mean (SD) & $1.08(0.19)$ \\
\hline
\end{tabular}

sented as percentages. All analyses were performed in SPSS version 24 software (IBM).

\section{Results}

\section{Patient characteristics}

The study population consisted of 32 patients presenting with typical CAD risk factor profiles, with a high percentage of diabetic or impaired glucose tolerance patients, positive history of myocardial infarction (MI) or $\mathrm{PCl}$, and suboptimal treatment of dyslipidemia. $68.8 \%$ of the patients were male, with a mean age of $68 \pm 8$ years. Most procedures were performed in stable coronary artery disease (SCAD), with 5 procedures performed in acute coronary syndromes (ACS).

\section{Procedure}

In most cases, the target lesion was located in the left anterior descending artery (68.75\%). About $60.0 \%$ of the procedures were performed using a transradial approach. The long-tapered BioMime Morph DES was successfully implanted in all 32 patients. Stents with a diameter of 3.0-2.5 mm (proximal to distal) were the most frequently used. We observed a need for deployment of an additional stent in $56 \%$ of the procedures (31\% proximal and $25 \%$ distal to the implanted stent), in $25 \%$ of cases due to proximal $(15.6 \%)$ or distal (9.4\%) dissection, or due to insufficient stent length in $31 \%$ of cases. Mean lesion length was $48 \mathrm{~mm}$, with a range of 35 to over $70 \mathrm{~mm}$. Stent to baseline minimal artery diameter ratio at the distal edge of lesion was $1.26 \pm 0.27$, and was similar in groups with single stent treatment $(1.30 \pm 0.29)$ and in patients who needed additional stent implantation (1.24 $\pm 0.26 ; p=0.60$ for $t$-test). Mean deployed to nominal stent diameter ratio at the distal edge was $0.90 \pm 0.13$. A GuideLiner catheter was used in $12.5 \%$ of procedures, while the buddy-wire technique was used in $9.4 \%$ of cases. Bifurcation lesions were treated in $40.6 \%$ of cases, with predominant anatomy in MEDINA classification 1,1,0. No major side branch, defined as greater than $1 \mathrm{~mm}$ diameter, was lost. A transradial approach was used in $60 \%$ of the $\mathrm{PCl}$ procedures. The long-tapered BioMime Morph drug-eluting stent (40-60 mm) was successfully implanted in all patients. Detailed characteristics of patients and procedures are presented in Table I. Stent implantation images and the QCA analysis prior to and post-stent implantation are presented in Table II and in Figures 1 and 2.

\section{Clinical follow-up}

During the 3-month follow-up period, 1 (3.1\%) patient was readmitted due to the reoccurrence of symptoms, in whom angiographic progression proximally to the previously deployed stent was revealed. The patient required target lesion revascularization (TLR) and no other major 


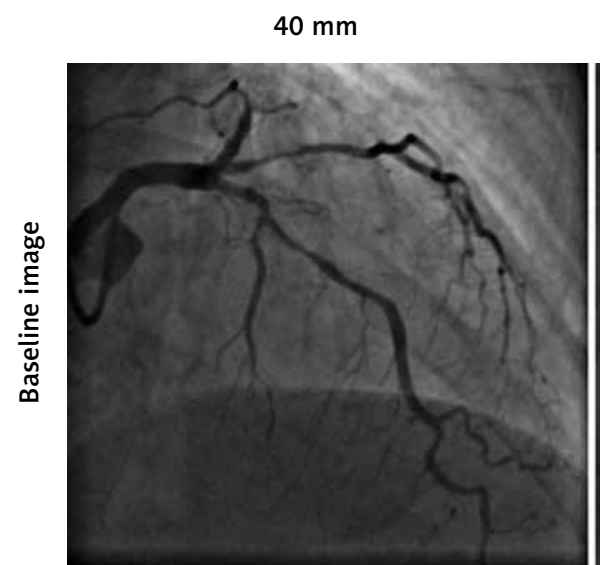

$50 \mathrm{~mm}$
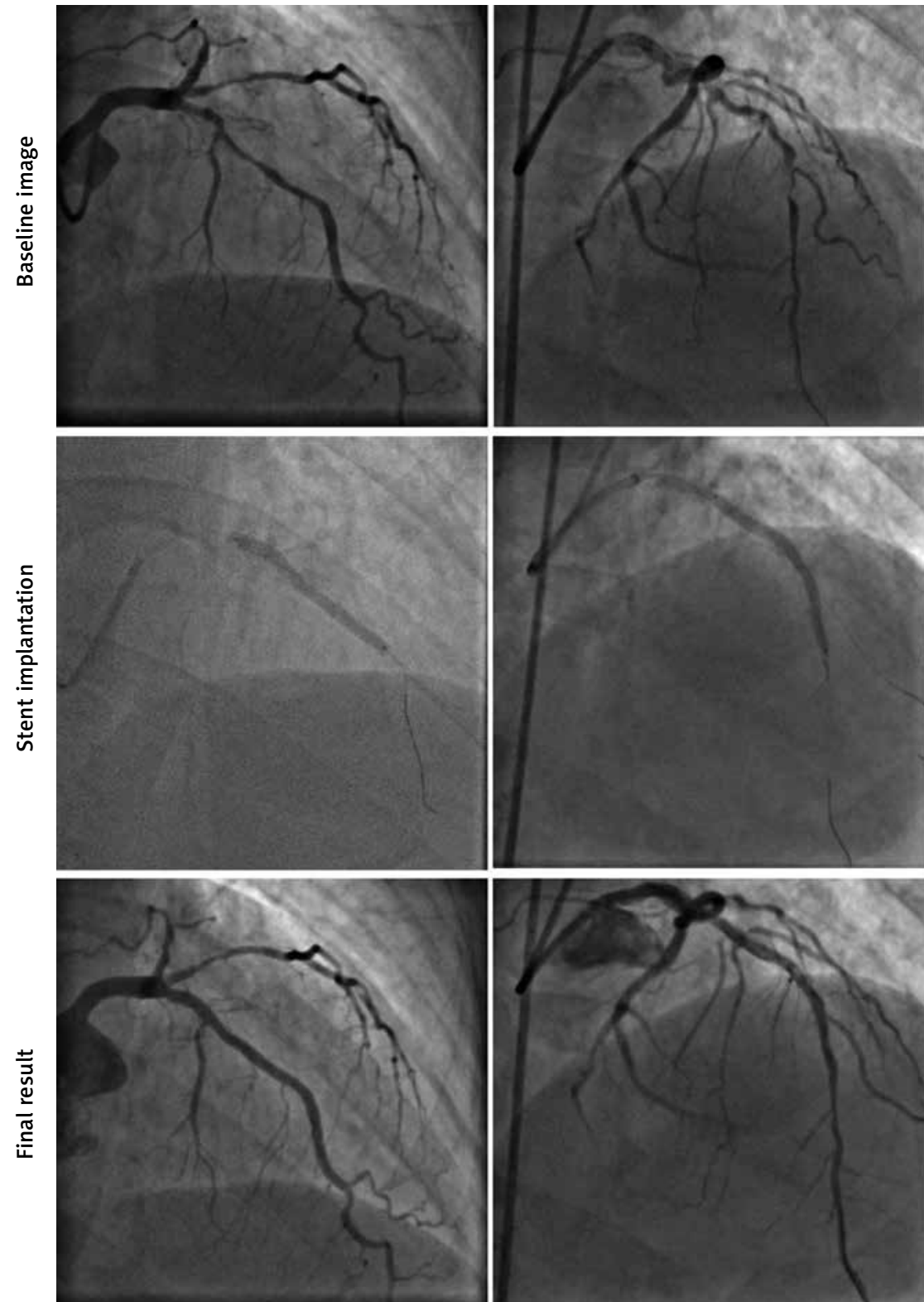
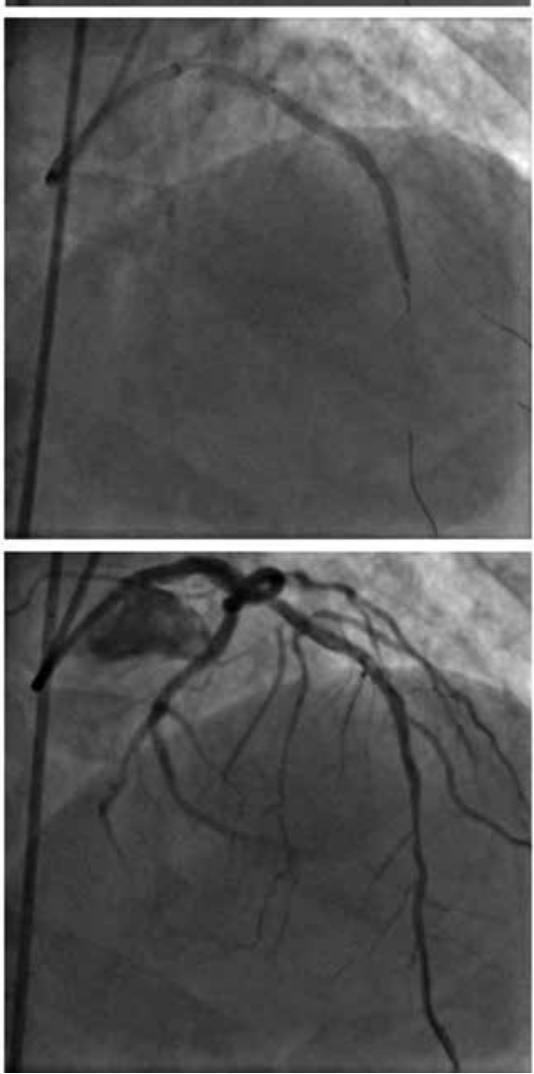

$60 \mathrm{~mm}$
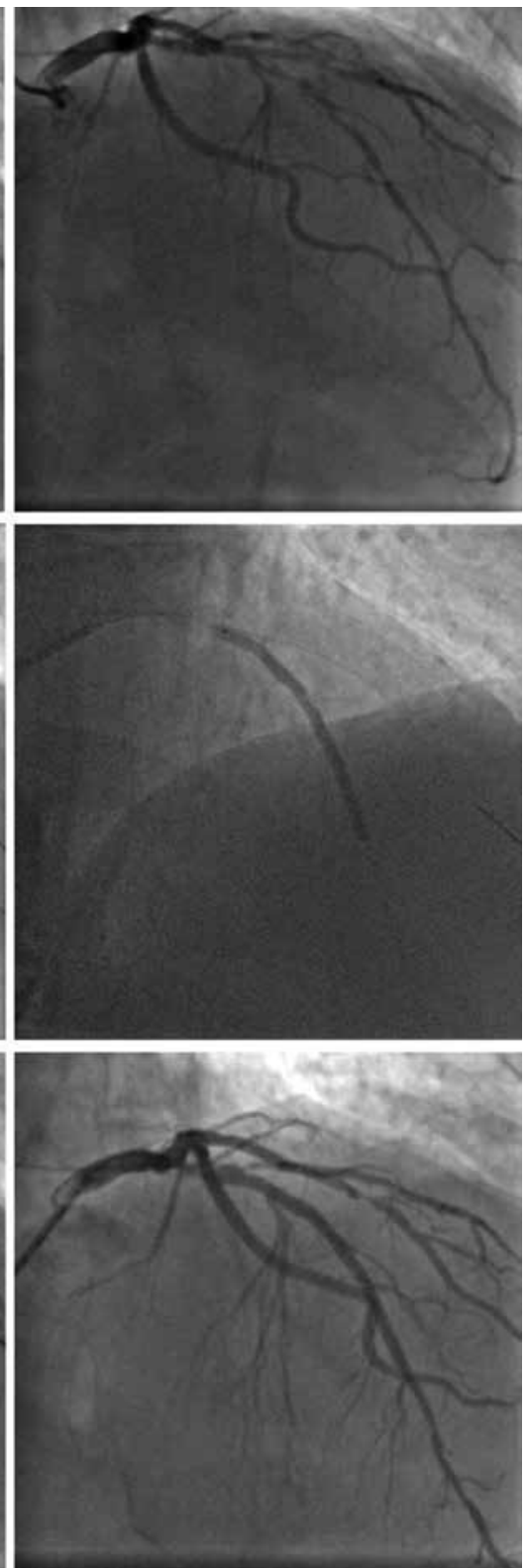

Figure 1. Long-tapered stent implantation $(40-60 \mathrm{~mm})$

adverse cardiac events (MACE) were observed. During the 6-month follow-up period no MACE were observed, although 1 patient was hospitalized for minor lower gastrointestinal bleeding. Data on 12-month follow-up were obtained from 30 patients. The TVR was observed in $1(3.3 \%)$ patient in the setting of stable CAD and another patient (3.3\%) experienced ACS with non-target vessel as the culprit lesion. One patient died 8 months after PCI due to multi-organ-failure (MOF) with no evidence of ACS as the primary cause.

\section{Discussion}

The new-generation DES have become a routine choice in the treatment of CAD. In current guidelines, full lesion coverage is the preferred $\mathrm{PCl}$ strategy [4]. Complete revascularization in this setting often requires using multiple stents in a single vessel, which is a direct consequence of the maximum stent lengths currently available. There is still a lack of data about the treatment of long diffuse coronary lesions using the single or multiple stent strategies.

Many researchers have investigated the problem of stented segment length, demonstrating that longer stent lengths are associated with a significantly higher risk of stent thrombosis and major coronary events [5-8]. Since the length of the segment requiring $\mathrm{PCl}$ remains a non-addressable issue, we tried to assess the impact of overlap reduction. It has been shown that stent over- 

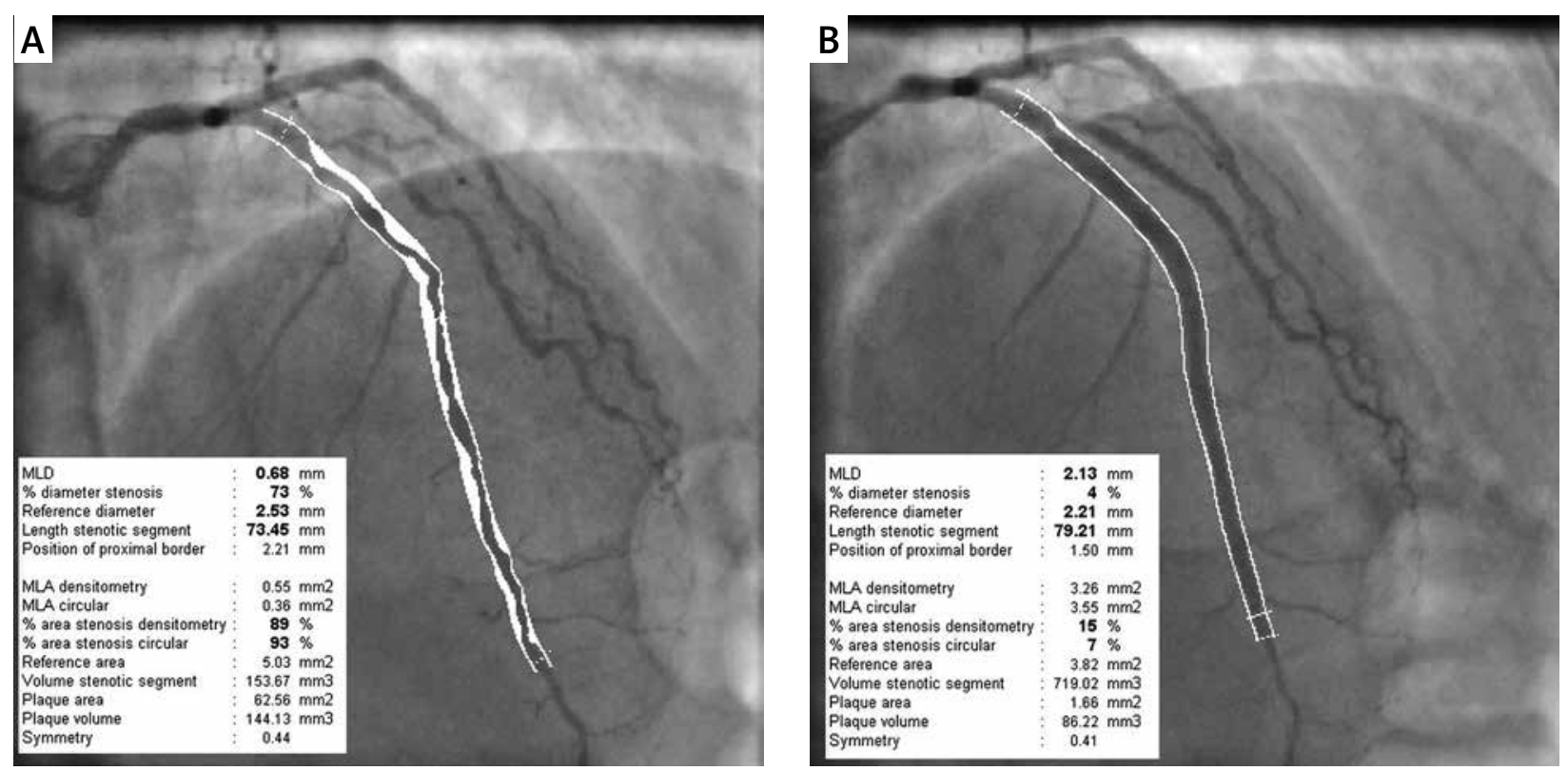

Figure 2. Quantitative coronary analysis (QCA) of lesion before (A) and after $\mathrm{PCl}(\mathbf{B})$

lap may be associated with impaired endothelialization. Increased strut density imposes a greater risk of side branch compromise, and additionally increases the risk of local stent fractures and arterial wall injury [9]. Given these factors, stent overlap compared with no overlap is associated with impaired clinical and angiographic outcomes during long-term follow-up [8, 10]. Moreover, standard new-generation DES hold their diameter with the stent length, which in cases of very long lesions may be a cause of stent size mismatch with the distal coronary lumen [11]. Using a long single-tapered DES addresses these aforementioned issues, theoretically giving a reduced risk of complications.

In our experience, device implantation appears to be efficacious independently of clinical presentation [12, 13]. In our case series, an all comers population was treated. We successfully deployed the stent in $100 \%$ of the procedures. However, long lesion angioplasty has a significant learning curve, especially when diffused atherosclerosis is present, and operator experience is of tremendous significance.

We observed one TVR in the 3-month follow-up, and another one on 12-month follow-up, with one death (due to MOF) and no major bleeding. Furthermore, no other MACE were seen at 12 months, which in our opinion is an encouraging result.

One should note a need for additional stent implantation in $56 \%$ of the procedures, in $25 \%$ of cases due to dissection presence (both proximal or distal) and in the remaining $31 \%$ of cases due to insufficient length of the first implanted stent. The latter was probably due to a tendency for the operator to underestimate the lesion length or occurrence of unanticipated problems with long-stent insertion into the distal part of the vessel. Given the good deliverability of the device, even if in some cases "buddy wire" technique or extension catheter was needed, it might suggest the use of longer, even $60 \mathrm{~mm}$ tapered stents, to avoid overlap in the future. In addition, the expansion ratio of distal artery diameter, over 1.2 times compared to baseline, was associated with a low percentage of distal dissection, which was present in $15 \%$ of patients.

The notably low radial access rate, only in $60 \%$ of cases, might have been due to the anticipated complexity of procedure, caused by anatomy and length of lesions. However, there was a 100\% success rate of stent delivery in both femoral and radial access groups, irrespective of clinical setting. We used the GuideLiner catheter in only $12.5 \%$ of cases, while the buddy-wire technique was used in $9.4 \%$ of cases. In our opinion, these observations demonstrate acceptable deliverability of the device in an all comers population. Obviously, proper preparation of the lesion with high pressure inflations is crucial to facilitate stent delivery and decrease the need for additional technique application (e.g. "buddy wire" technique, extension microcatheter).

Contrast medium usage in our case series was similar to those in recently emerging clinical studies with long stents $[14,15]$. We hypothesize that a lower number of implanted stents should result in lower contrast medium and radiation use. However, there is still a lack of published data involving a direct comparison of multiple stenting versus the long single-stent strategy in terms of contrast expenditure or radiation exposure.

\section{Study limitations}

The present study included a relatively small, all-comers patient population and requires a larger cohort study for replication of these results. A second limitation is the 
lack of a control group to compare the use of contrast medium and radiation exposure.

\section{Conclusions}

The long sirolimus-eluting stent with tapered structure was characterized by good deliverability in long coronary lesions, although in some cases "buddy wire" or extension microcatheter use was necessary. Previous lesion preparation even with a non-compliant balloon is important for successful stent introduction. Operator experience in complex $\mathrm{PCl}$ plays an important role. Follow-up at 3, 6, and 12 months showed no significant major adverse cardiovascular events associated with the device. Further investigational clinical trials are needed to compare multiple vs. single-stent strategies in patients with long coronary lesions.

\section{Conflict of interest}

The authors declare no conflict of interest.

\section{References}

1. Piepoli MF, Hoes AW, Agewall S, et al. 2016 European Guidelines on cardiovascular disease prevention in clinical practice. Eur Heart J 2016; 37: 2315-81.

2. Podolec J, Kopec G, Niewiara L, et al. Chemokine RANTES is increased at early stages of coronary artery disease. J Physiol Pharmacol 2016; 67: 321-8.

3. Podolec J, Niewiara L, Skiba D, et al. Higher levels of circulating naïve CD8 + CD45RA + cells are associated with lower extent of coronary atherosclerosis and vascular dysfunction. Int I Cardiol 2018; 259: 26-30.

4. Kolh P, Windecker S, Alfonso F, et al. 2014 ESC/EACTS Guidelines on myocardial revascularization. Eur J Cardiothoracic Surg 2014; 46: 517-92.

5. Aoki J, Ong ATL, Rodriguez Granillo GA, et al. "Full metal jacket" (stented length > or $=64 \mathrm{~mm}$ ) using drug-eluting stents for de novo coronary artery lesions. Am Heart J 2005; 150: 994-9.

6. Ruchin PE, Trabattoni D, Fabbiocchi F, et al. Use of multiple overlapping sirolimus-eluting stents for treatment of long coronary artery lesions: results from a single-center registry in 318 consecutive patients. Int J Cardiol 2009; 134: 231-7.

7. Caputo RP, Goel A, Pencina M, et al. Impact of drug eluting stent length on outcomes of percutaneous coronary intervention (from the EVENT Registry). Am I Cardiol 2012; 110: 350-5.

8. Räber L, Jüni P, Löffel L, et al. Impact of stent overlap on angiographic and long-term clinical outcome in patients undergoing drug-eluting stent implantation. J Am Coll Cardiol 2010; 55: 1178-88.

9. Lim SY, Jeong MH, Hong SJ, et al. Inflammation and delayed endothelization with overlapping drug-eluting stents in a porcine model of in-stent restenosis. Circ J 2008; 72: 463-8.

10. Degertekin M, Arampatzis CA, Lemos PA, et al. Very long sirolimus-eluting stent implantation for de novo coronary lesions. Am J Cardiol 2004; 93: 826-9.

11. Timmins LH, Meyer CA, Moreno MR, Moore JE. Mechanical modeling of stents deployed in tapered arteries. Ann Biomed Eng 2008; 36: 2042-50.
12. Podolec J, Niewiara $Ł$, Baran J, et al. First in Poland, unique 60$\mathrm{mm}$ long single drug eluting tapered stent implantation in a patient with unstable angina. Kardiol Pol 2017; 75: 78.

13. Podolec J, Baran J, Niewiara $Ł$, et al. Intravascular ultrasonography guided 60-mm long drug-eluting tapered stent implantation in a long calcified lesion in a patient with stable coronary artery disease. Adv Interv Cardiol 2017; 13: 88-9.

14. Valero E, Consuegra-Sánchez L, Miñana G, et al. Initial experience with the novel BioMime $60 \mathrm{~mm}$-long sirolimus-eluting tapered stent system in long coronary lesions. Eurointervention 2018; 13: 1591-4.

15. Premchand RK, Kumar YS. A report of successful procedural, clinical, and angiographic outcomes with a tapered stent of a patient in naturally tapered coronary vessel. J Clin Diagn Res 2017; 11: OD06-7. 